\title{
Master-equation approach to understanding multistate phase-change memories and processors
}

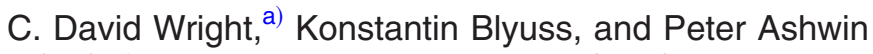 \\ School of Engineering, Computer Science and Mathematics, University of Exeter, Exeter EX4 4QF, \\ United Kingdom
}

(Received 6 October 2006; accepted 15 January 2007; published online 9 February 2007)

\begin{abstract}
A master-equation approach is used to perform dynamic modeling of phase-transformation processes that define the operating regimes and performance attributes of electronic (and optical) processors and multistate memory devices based on phase-change materials. The predictions of the so-called energy accumulation and direct-overwrite regimes, prerequisites for processing and memory functions, respectively, emerge in detail from the model, providing a theoretical framework for future device design and evaluation. (c) 2007 American Institute of Physics.
\end{abstract}

[DOI: $10.1063 / 1.2475606]$

Electrical memory devices based on the reversible transition between amorphous and crystalline phases in chalcogenide alloys, such as GeSbTe, are attracting much interest, in particular, as possible replacements for silicon "flash" memory. ${ }^{1,2}$ The development of binary memories currently predominates, but multistate memories will be of much interest in future since they offer greater storage capacity. More remarkable and far-reaching potential applications of phase-change technology, recently discussed by Ovshinsky and Pashmakov ${ }^{3}$ and Ovshinsky ${ }^{4}$ include the provision of non-Von-Neumann (micro) processing devices capable of both general-purpose computation and "cognitive" function. The origins of such possibilities lie in the detail of the phasetransformation event itself. In conventional phase-change memories crystallization relies on both electronic and thermal effects; applying a voltage above a certain value induces a conducting on state in the previously high-resistance amorphous material, allowing current to flow which in turn generates heat to drive crystallization. The electrical resistance during switching changes abruptly at the "percolation threshold," where growing (nano)cystallites merge to form the first conducting pathways between device electrodes. It is the prethreshold region that offers the potential to perform generalpurpose computation and provides artificial neuronlike capabilities. This may be explained by considering prepercolation behavior to involve energy accumulation; energy is accumulated and crystal clusters grow as each input pulse is applied and when enough energy has been accumulated to reach the percolation threshold the cell resistance changes abruptly. This energy accumulation property has the potential to implement basic mathematical operations such as addition, subtraction, multiplication, and division, as well as more complex functions such as factoring, encryption, and logic. . $^{3,4}$ The accumulation property, the presence of a distinct threshold, and a nonlinear (output) transition (between resistance states) mimic the basic action of a biological neuron. Furthermore, the (synaptic) weighting of inputs might be provided by another phase-change cell operating in the multilevel storage regime. Multilevel storage has already been demonstrated for both optical and electrical memories., Thus, an artificial neuron might be achieved using only

\footnotetext{
${ }^{a)}$ Electronic mail: david.wright@exeter.ac.uk
}

phase-change cells, operating in the energy-accumulating regime to mimic the operation of the neuronal body and the multilevel storage regime to mimic synaptic weighting.

Several methods have been used over the years to model the process of crystallization in phase-change alloys, such as the Johnson-Mehl-Avrami-Kolmogorov (JMAK) model. Unfortunately many of the assumptions on which JMAK is based are violated in real switching events in phase-change devices. Furthermore JMAK cannot distinguish materials with the same crystallized fraction but different crystallite size distributions, and this is important to predict the dynamical progress of the complex annealings necessary for multistate memories and phase-change processors. Another common approach is based on separable nucleation and growth models, often used to examine optical phase-change recording. ${ }^{5}$ However, such approaches deal only with crystal clusters at or above the critical (stable) size, whereas subcritical clusters are also likely to play a significant role in the nanoscale dynamic behavior of future devices. An attractive, physically plausible, alternative to these more established methods is the master-equation approach that models the evolution of the crystal cluster size distribution during the entire phase-transformation process. ${ }^{6,7}$ Using rate equations, the frequencies of attachment and detachment of material "monomers" representing unit changes in crystal cluster sizes can be determined. We have investigated the use of both discrete ${ }^{6}$ and continuous ${ }^{7}$ versions of the master equation to model phase transformations in $\mathrm{Ge}_{2} \mathrm{Sb}_{2} \mathrm{Te}_{5}$. The work so far has concentrated on simulating bulk behavior. In this letter the master-equation approach is used to understand and to predict the detail of the phase-transformation events that lie behind the multistate memory and phase-change processor devices introduced above.

The basis of the master-equation approach is the determination of the distribution function $Z(n, t)$ representing the density of crystal clusters of size $n$ (monomers) at moment $t$. Changes in the crystal cluster sizes occur when monomers are attached to or detached from existing clusters. In all the simulations presented here, a discrete version of the master equation has been used for which the temporal evolution of the cluster density $Z$ is given by ${ }^{6}$ 


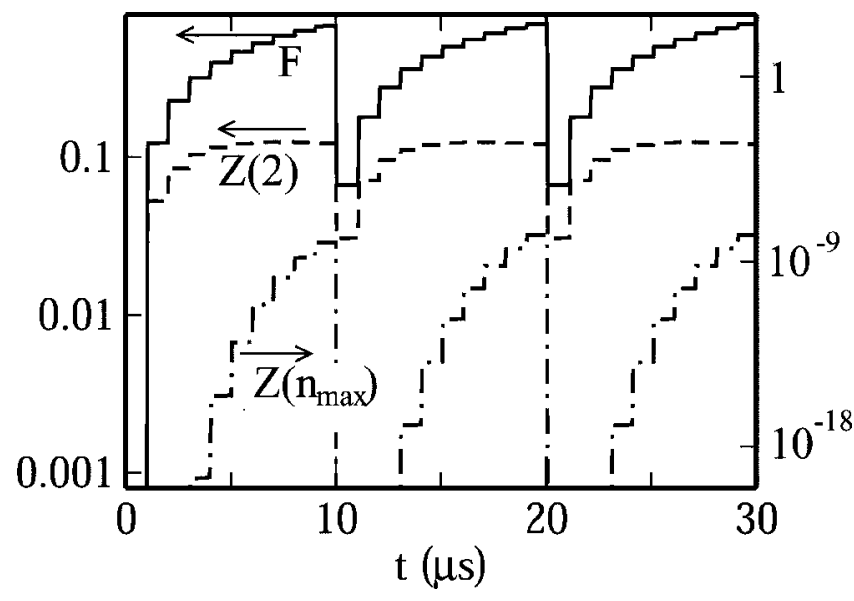

FIG. 1. Crystallized fraction $F$ [i.e., sum of $Z(n)$ from $n=2$ to $n=n_{\max }$ ] (solid), density of dimers $Z(2)$ (dashed), and density of multimers $Z\left(n_{\max }\right)$ (dash-dot) for an annealing cycle comprising successive $350{ }^{\circ} \mathrm{C}, 50 \mathrm{~ns}$ pulses at $1 \mu$ s intervals, followed by a reset (amorphization) pulse.

$$
\begin{aligned}
\frac{\partial Z(n, t)}{\partial t}= & f(n-1, t, T) Z(n-1, t)+d(n+1, t, T) Z(n+1, t) \\
& -f(n, t, T) Z(n, t)-d(n, t, T) Z(n, t)
\end{aligned}
$$

where $f(n, t, T)$ and $d(n, t, T)$ are, respectively, the rates of attachment to and detachment from a crystal cluster of size $n$ units (monomers) and depend strongly on temperature $T$ [primarily via (i) Boltzmann factors defined in terms of Gibbs free energy differences between cluster sizes $n$ and $n+1$ and (ii) a jump frequency at the crystal/amorphous interface]. Our model is discussed in detail elsewhere; ${ }^{6,7}$ here we are primarily concerned with its application to understanding new device behavior. In solving the master equation it is often assumed that the system undergoes phase transformations while staying close to a quasiequilibrium. This approximation allows expression of the detachment frequencies through the attachment frequencies and a quasiequilibrium cluster size distribution. ${ }^{6}$ Here, the attachment frequency is assumed to be proportional to the amount of free monomer while the detachment frequency is assumed to be independent of this.

Equation (1) is a coupled system of ordinary differential equations that can be solved using the Fortran numerical algorithms group routine D02EJF, a variable-order, variablestep method implementing a backward-differentiation formula suitable for a stiff system of first-order ordinary differential equations. Simulations were performed for a relatively small range of cluster sizes due to the stiffness of the equations; essentially we assign the maximum cluster size for stable numerical simulation over the range of temperatures used for each annealing. All the results presented here are for the commonly used $\mathrm{Ge}_{2} \mathrm{Sb}_{2} \mathrm{Te}_{5}$ alloy. The values of the material parameters necessary for the evaluation of Eq. (1) are as described in detail elsewhere ${ }^{6}$ with the exception of the activation energy (here assumed to be $2.1 \mathrm{eV}$ ), the interfacial energy density between phases (here taken to be $0.075 \mathrm{~J} \mathrm{~m}^{-2}$ ), the cap angle for growing crystallites (here set to $70^{\circ}$ ), and the viscosity prefactor (here taken to be $8 \times 10^{-16} \mathrm{~Pa} \mathrm{~s}$ )

Figure 1 shows the result of the simulation when the initially amorphous cell (all monomers) is subject to successive annealing pulses. Each pulse was simulated by setting

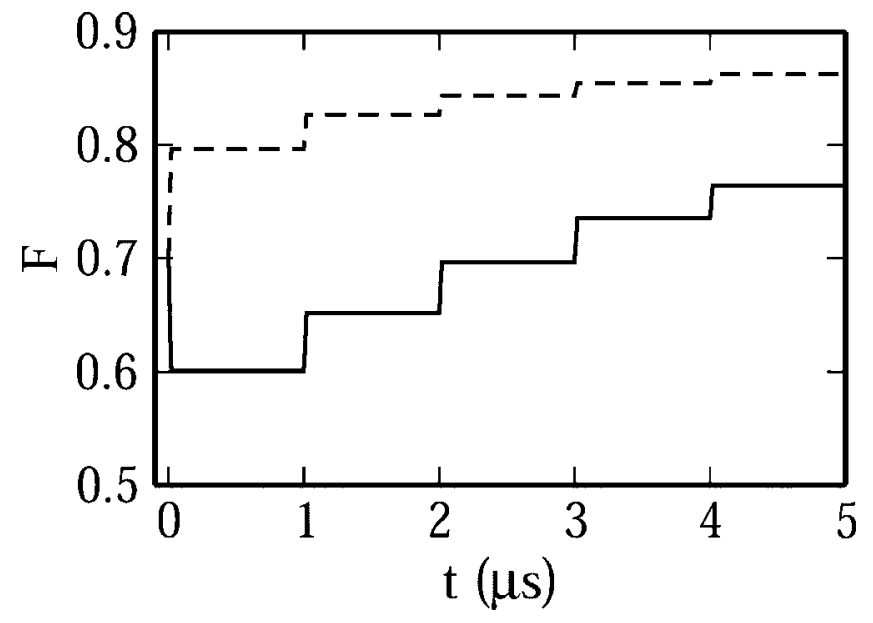

FIG. 2. Temporal evolution of the crystallized fraction $F$ for different initial cluster size distributions, in which a $70 \%$ crystallized starting material consists entirely of dimers (solid) or multimers (dashed). Annealing cycle consisted of $20 \mathrm{~ns}, 450{ }^{\circ} \mathrm{C}$ pulses applied at $1 \mu$ s intervals. The $20 \mathrm{~ns}$ pulses are used here to capture the strikingly different dynamic behavior that can occur; differences are still present in this case with longer pulses, but are not so marked.

the temperature in the cell to $350{ }^{\circ} \mathrm{C}$ for $50 \mathrm{~ns}$; pulses were $1 \mu \mathrm{s}$ apart. The cell was reset by the tenth pulse into the amorphous state. In reality voltage or current pulses would be applied to the phase-change cell and heating would be due to a combination of electrical and thermal effects, ${ }^{3,4,8}$ but the origin of the heating is not considered here, merely its effects on crystallization behavior. Figure 1 reveals a monotonically increasing crystallized fraction with increasing number of annealing pulses. The number of monomers (not shown) decreases monotonically while the number of dimers $(n=2)$ and multimers $\left(n=n_{\max }\right)$ increases monotonically through annealing. The phase-change cell is effectively accumulating energy with each pulse and crystal clusters grow in size and number as the annealing progresses. Each particular crystallized fraction in the annealing cycle corresponds to a particular cell resistance in a real device, and so by monitoring the cell resistance a decimal counter/adder could easily be obtained. Indeed, to make the process of decimal addition even simpler the device could be operated in threshold mode, where the completion of a count to ten is designed to coincide with crossing the percolation threshold. For spherical clusters the percolation threshold occurs theoretically at a $1 / 3$ crystal fraction. It would be a simple matter to change the annealing regime for the percolation threshold to be reached after ten pulses.

Figure 1 also reveals indirectly one of the main advantages of the master-equation approach in that it allows modeling of the dynamic evolution of crystal cluster populations, not just an aggregated crystallized fraction as in the JMAK approach. Indeed a JMAK simulation may potentially generate erroneous results, as illustrated by Fig. 2. Here the result of a five-step annealing process from an initial $70 \%$ crystal fraction for two very different initial cluster size distributions is shown. In the case when initially all crystals are dimers the first annealing pulse leads to a reduction in the crystal fraction, since it is energetically favorable for some dimers to lose monomers to become monomers (amorphous) themselves. For a material initially consisting entirely of multimers, however, the crystallized fraction grows monotonically. This strikingly different dynamic behavior reiterates the imAIP license or copyright; see http://apl.aip.org/apl/copyright.jsp 


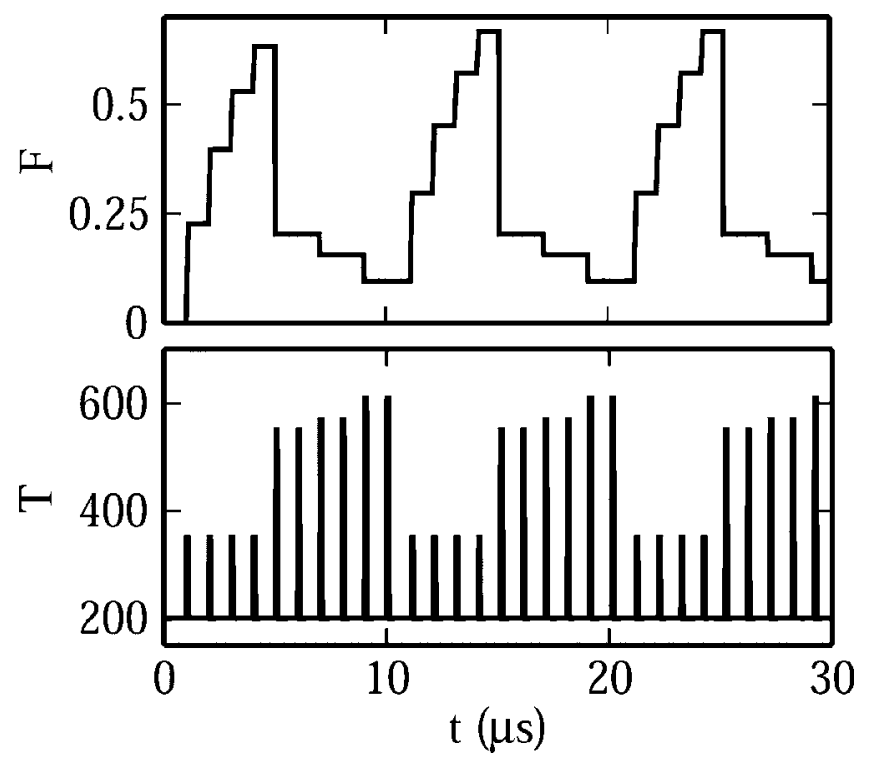

FIG. 3. Crystalline fraction (top) as a function of time during an annealing comprising of $100 \mathrm{~ns}$ pulses at temperatures of $350{ }^{\circ} \mathrm{C}$ (four pulses), $550{ }^{\circ} \mathrm{C}$ (two pulses), $570{ }^{\circ} \mathrm{C}$ (two pulses), and $610^{\circ} \mathrm{C}$ (two pulses).

portance of correctly understanding the initial conditions (cluster size distribution).

Next, we explore the "direct-overwrite" regime, ${ }^{3,4}$ where at a higher temperature the material undergoes a transformation to a state that may be between crystalline and amorphous. Figure 3 shows the results of simulations together with details of temperature variation during annealing. For relatively low temperature annealings, the crystalline fraction is "remembered" from the previous step (crystallized fraction due to each successive pulse adds to preexisting state) and we are in the energy accumulation regime, while for higher temperatures the crystalline fraction moves repeatably within the space of one pulse directly to a given state. In this latter so-called multistate or direct-overwrite regime, the final crystallized fraction is independent of the initial state and depends only on the annealing pulse duration and amplitude (temperature), thus illustrating the possibility of switching between successive crystallization states without complete resetting. Indeed, at least 16 separate states have been programed into real devices using this technique. ., $^{3}$

The two distinct regimes, energy accumulation and direct overwrite, revealed by Figs. 1 and 3 are often determined for real devices by measuring cell resistance as a function of write current, starting in the amorphous phase. This results in a characteristic " $U$ " curve that is most helpful in device evaluation and design; the left-hand side of the $U$ curve is the energy-accumulation regime while the righthand side is the direct-overwrite region. ${ }^{3,4}$ This characteristic U curve may also be predicted theoretically using the masterequation approach. Specimen results are shown in Fig. 4, where the effect on cell resistivity (calculated from the crystal fraction using Bruggeman effective medium theory ${ }^{9,10}$ ) of temperature pulses of different amplitudes and durations is shown; the characteristic U shape is clearly revealed.

In summary the master-equation approach has proven extremely valuable for understanding the detailed phasetransition behavior, including dynamic effects, of phasechange materials for current and, importantly, possible future devices that demonstrate computational processing and mul-

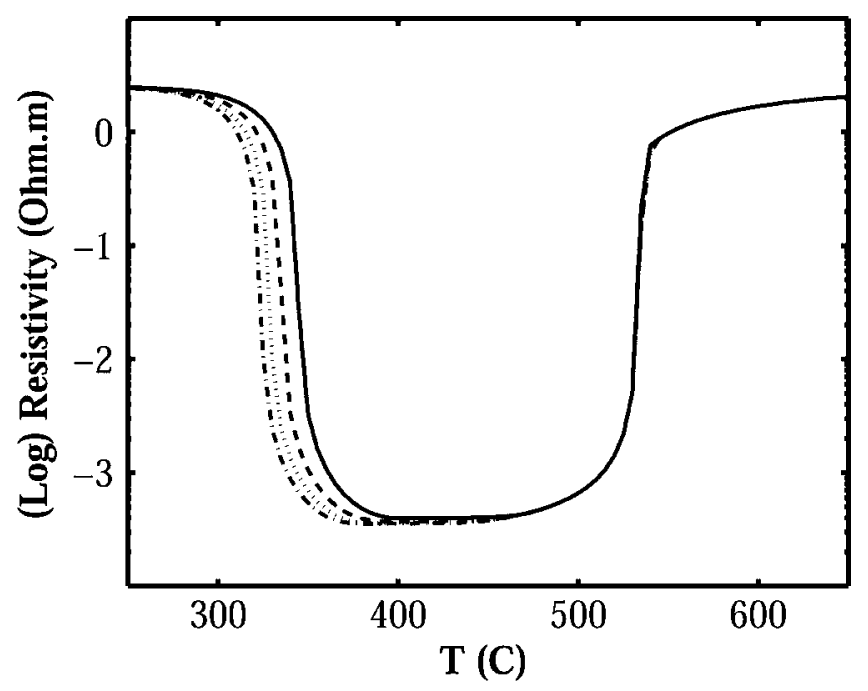

FIG. 4. Final resistivity of phase-change cell as a function of the temperature of annealing pulses of duration of $200 \mathrm{~ns}$ (solid line), $300 \mathrm{~ns}$ (dashed), $400 \mathrm{~ns}$ (dotted), and $500 \mathrm{~ns}$ (dash-dot). A U-shaped curve with an additive response at lower temperatures and a direct-overwrite response at higher temperatures is revealed. Room temperature conductivity of crystalline and amorphous phases assumed, respectively, to be 3250 and $0.4 \Omega^{-1} \mathrm{~m}^{-1}$.

tistate storage capabilities. The model successfully predicts that both crystallization and amorphization (partial or complete) can be observed, dependent on the duration and temperature used for annealing, leading to two distinct operating regimes-energy accumulation and direct overwrite. The results are extremely sensitive to the annealing used, and suggest that tight control will be necessary to ensure that a desired level of partial crystallization is achieved in real devices. The model as implemented here has a number of limitations. Firstly, only temporal, not spatial, dynamics of cluster formation are considered. This can be overcome since space dependence can be introduced via the temperature distribution; ${ }^{7}$ hence real device geometries can be modeled. Secondly, for practical computation the maximum cluster size is limited by stiffness of the equations. Simulations presented here use a maximum cluster size in the range of 50100 monomers, corresponding to cluster sizes around $5 \mathrm{~nm}$ (for $\mathrm{Ge}_{2} \mathrm{Sb}_{2} \mathrm{Te}_{5}$ ). Such a size limitation should be less important if spatial dependence is introduced and the simulation space split into nanoscale-sized cells.

One of the authors (C.D.W.) acknowledges support jfrom the Leverhulme Trust via its Fellowship scheme. The EPSRC is also acknowledged for its support via Grant GR/ S31662/01.

${ }^{1}$ M. Wuttig, Nat. Mater. 4, 265 (2005).

${ }^{2}$ A. L. Greer and N. Mathur, Nature (London) 437, 1246 (2005).

${ }^{3}$ S. R. Ovshinsky and B. Pashmakov, Mater. Res. Soc. Symp. Proc. 803, 49 (2004).

${ }^{4}$ S. R. Ovshinsky, Jpn. J. Appl. Phys., Part 1 43, 4695 (2004).

${ }^{5}$ B. Hyot, V. Gehanno, B. Rolland, A. Fargeix, C. Vannufel, F. Charlet, B. Bechevet, J. M. Bruneau, and J. P. Desre, J. Magn. Soc. Jpn. 25, 414 (2001).

${ }^{6}$ S. Senkader and C. D. Wright, J. Appl. Phys. 95, 504 (2004).

${ }^{7}$ K. B. Blyuss, P. Ashwin, A. P. Bassom, and C. D. Wright, Phys. Rev. E 72, 011607 (2005).

${ }^{8}$ C. D. Wright, M. Armand, and M. M. Aziz, IEEE Trans. Nanotechnol. 5, 50 (2006).

${ }^{9}$ D.-H. Kim, F. Merget, M. Laurenzis, P. H. Bolivar, and H. Kurz, J. Appl. Phys. 97, 083538 (2005).

${ }^{10}$ M. M. Aziz and C. D. Wright, J. Appl. Phys. 97, 103537 (2005). 